\title{
LVIII. Dissymmetry in the electrolytic discharge
}

\section{Alfred Tribe F.Inst.C.}

To cite this article: Alfred Tribe F.Inst.C. (1883) LVIII. Dissymmetry in the electrolytic discharge , Philosophical Magazine Series 5, 15:96, 391-396, DOI: 10.1080/14786448308627372

To link to this article: http://dx.doi.org/10.1080/14786448308627372

曲 Published online: 28 Apr 2009.

Submit your article to this journal 준

Џll Article views: 2

Q View related articles $₫$ 
values of $\int_{\delta=180^{\circ}}^{\delta=0^{\circ}} \cos \delta-\cos \alpha$ corresponding to each value of $\frac{\cos \delta-\cos \alpha}{\sin \alpha}$ were calculated, until each increment of $\int_{\delta=180^{\circ}}^{\delta=0^{\circ}} \cos \delta-\cos \alpha$ became very small as compared with each increment of $\frac{\cos \delta-\cos \alpha}{\sin \alpha}$, it would be found that the different values of $\int_{\delta=180^{\circ}}^{\delta=0^{\circ}} \cos \delta-\cos \alpha$ form the ordinates of a curve which approaches towards an asymptote,--the abscissæ representing the current-strengths, and the ordinates the amount

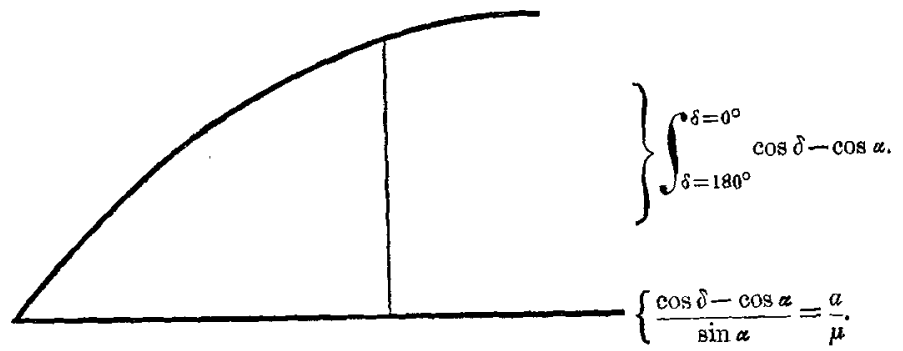

of magnetism of a magnetic circle of molecules-that is, the intensity of magnetism of the iron.

LVIII. Dissymmetry in the Electrolytic Discharge. By ALFRED TrIBE, F.Inst.C., Lecturer on Chemistry in Dulwich College*.

IN the Philosophical Magazine for June 1881, p. 446, I 1 gave the results of some experiments, from which it was concluded that the electrical discharge through an electrolyte whose transverse section was greater than the width of the electrodes was accompanied by dissymmetry in opposite but corresponding parts of the field of electrolytic action. The evidence consisted in the very different superficial magnitudes of the electrifications registered on similar plates of silver in corresponding positions near the + and - electrodes respectively. The silver plates, which I have called analyzers, were immersed in a solution of copper sulphate undergoing electrolysis. It was likewise shown that the dissymmetry was connected with the spreading-out of the lines of force; for as this was prevented, so the dissymmetry was less and less marked;

* Communicated by the Author. 
and when the lines crossed the field parallel to one another, what I have regarded as evidence of dissymmetry was no longer observed.

I had hoped to work at several questions suggested by these results; but as $I$ am unable at present to proceed with the experimental development of the investigation, I propose in this communication to record without further delay my unpublished observations bearing more especially on the subject of dissymmetry. This is the more advisable, in view of the interesting observations of Prof. Roiti (Nuovo Cimento, s. 3. vol. x.), and of the recent experimental and theoretical studies of Drs. Pasquilini and Volterra (A. dell' Accad. Sc. Torino, vol. xviii.).

In the first set of experiments to be described I employed a trough $305 \times 305$ millim. and 128 millim. deep, a 5-per-cent. solution of copper sulphate filling the trough to within 8 millim. of the top; copper electrodes about 7.5 millim. wide, placed in grooves running down the centre of two opposite sides of the vessel; analyzers of fine silver, $67 \times 7$ millim. (weighing about 75 gram), placed lengthwise, midway between the surface of the solution and the bottom of the cell, and parallel with a vertical plane joining the electrodes. The analyzers were supported by means of a small wedge of the metal projecting from the lower edge at the centre of the plates, this being fixed into a small stand of paraffined wood. In each experiment one analyzer was employed and a current of two ampères for six minutes.

The numerical results of twelve experiments are given in the annexed horizontal section (fig. 1), showing the position occupied by the several analyzers. It will be remembered that the + ion (copper) and the peroxide of silver form permanent images of the - and + electrifications respectively, so that after the completion of an experiment the magnitudes of these electrifications can be measured. It will also be remembered that the + ion is deposited on that end of the analyzer nearest the + electrode, or the side of the cell in which this electrode is fixed, and that the grey silver peroxide is formed on that part of the analyzer nearest the electrode, the two effects being separated by a part of the plate on which no visible action has taken place. The numbers give the lengths of the electrifications in millimetres.

In the cases where they were the same on the two sides of the analyzer, numbers are placed on the one side only in the diagram. Of course the numbers at the ends nearest the + electrode show -, while those at the ends nearest the - electrode refer to + electrifications. 
Fig. 1.

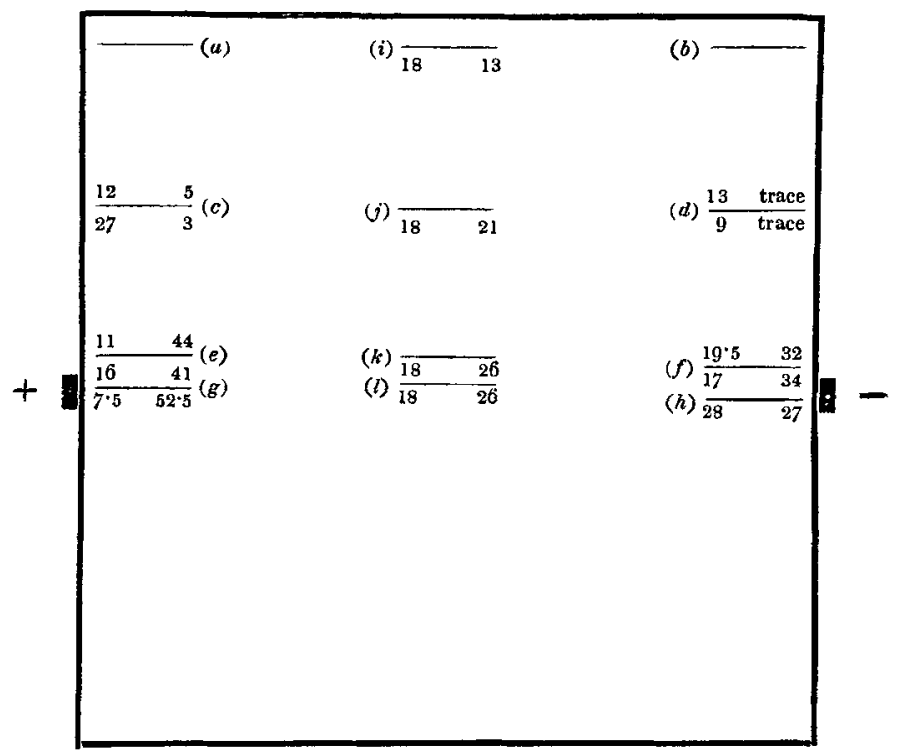

The electrochemical actions on the analyzers $a, b$ were too small to allow of a definite opinion being formed as to dissymmetry in the parts of the field surrounding them; but in the parts of the field $c, d, e, f$, and $g, h$, dissymmetry was most distinctly indicated by differences in the magnitudes of the electrification of the same sign on the several analyzers in opposite but corresponding positions. Taking the corresponding parts of the field where the greatest dissymmetry would appear to exist, the negative electrifications on $g$ and $h$ were in the proportion of $1: 3 \cdot 6$, while the + on the same plates were as $1 \cdot 9: 1$.

In connexion with these experiments, I would note one or two other observations not directly connected with dissymmetry. The relation which I have already shown to exist (Proc. Roy. Soc. 1881, p. 435) between the shape of the boundaries of the electrochemical deposits (i.e. where the chemical effects of the electrifications meet the unaffected surface of the analyzer) enables the directions of the lines of force to be determined in the several parts of an electrolytic field, unless it is very small in intensity. The lines across the field cut by the analyzers $g, l, h$ were thus shown to be parallel with the longer edges or sides of the silver plates; and in the immediate neighbourhood of $i, j$, and $k$ the lines were approximately in the same direction; but surrounding the plates $c, d, e$, 
and $f$ the curvilinear character of the lines was most distinctly shown to exist.

I would also direct attention to the magnitudes of the electrifications on the analyzers $i, j, k$. It will be observed that the - was the same on each of the plates, while the + varied from a lineal distribution of 13 to 26 millim. This would appear to show that the magnitudes at least of the - electrifications bear little or no relation to the intensity of the field, as it is obvious considerable variations in this respect existed in the several parts of the field. Any generalization, however, to be of real value would require many more observations on this branch of the subject.

Another point is the variations in the relative magnitudes of the opposite electrifications. In eight of the cases where the effects of both electrifications were sufficiently marked to allow of measurement, the + were greater on both or one side of the analyzer than were the - on the same side or sides; but in four cases the - were greater than the + .

Figs. 2 and 3 are diagrams of the plates $g$ and $e$ (fig. 1). The dotted lines indicate the shape of the boundaries, and the spaces enclosed between one of these lines and the corresponding end of the analyzer the magnitudes of the respective electrifications; $\alpha$ to $\beta$ being the,$- \beta$ to $\gamma$ the part of nonelectrification, and $\gamma$ to $\delta$ the + .

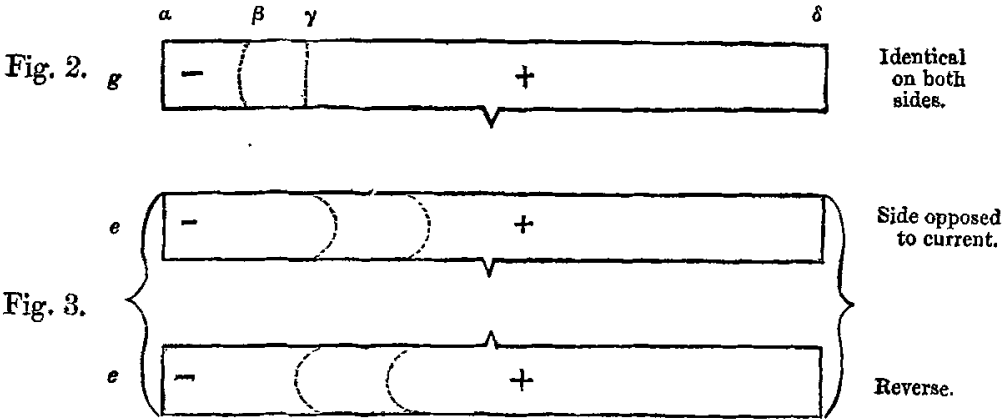

II. Dissymmetry by Interferenee.-I now describe some experiments made in the hope of throwing light on the subject of this paper. The results I do not offer as an explanation. I record them in this place, first, for the interest which belongs to them apart from other considerations; secondly, they may perhaps serve as the starting-point for fresh inquiry.

A trough was employed 305 millim. long, 120 broad, and 128 deep ; and copper electrodes of the b readth and depth of the cell placed at its respective ends. The strength of copper- 
sulphate solution, dimensions \&c. of analyzer, were the same as used in the previous experiments; but a current of one ampère was employed.

An ebonite plate $76 \times 76$ millim., about $1 \frac{1}{2}$ millim. in thickness, was placed parallel to the electrodes and in the centre of the liquid. In the position $A$ shown in the vertical section (fig. 4) it was midway between the electrodes; in $\mathrm{B}, 71$ millim. from the + electrode; and in C, 71 millim. from the electrode. Analyzers $a, b, c, d, e, f$ were successively placed lengthwise 2 millim. from, and perpendicular to, the centre of the plates.

The numbers in the diagram refer, as before, to the electrifications on the several analyzers.

Fig. 4.

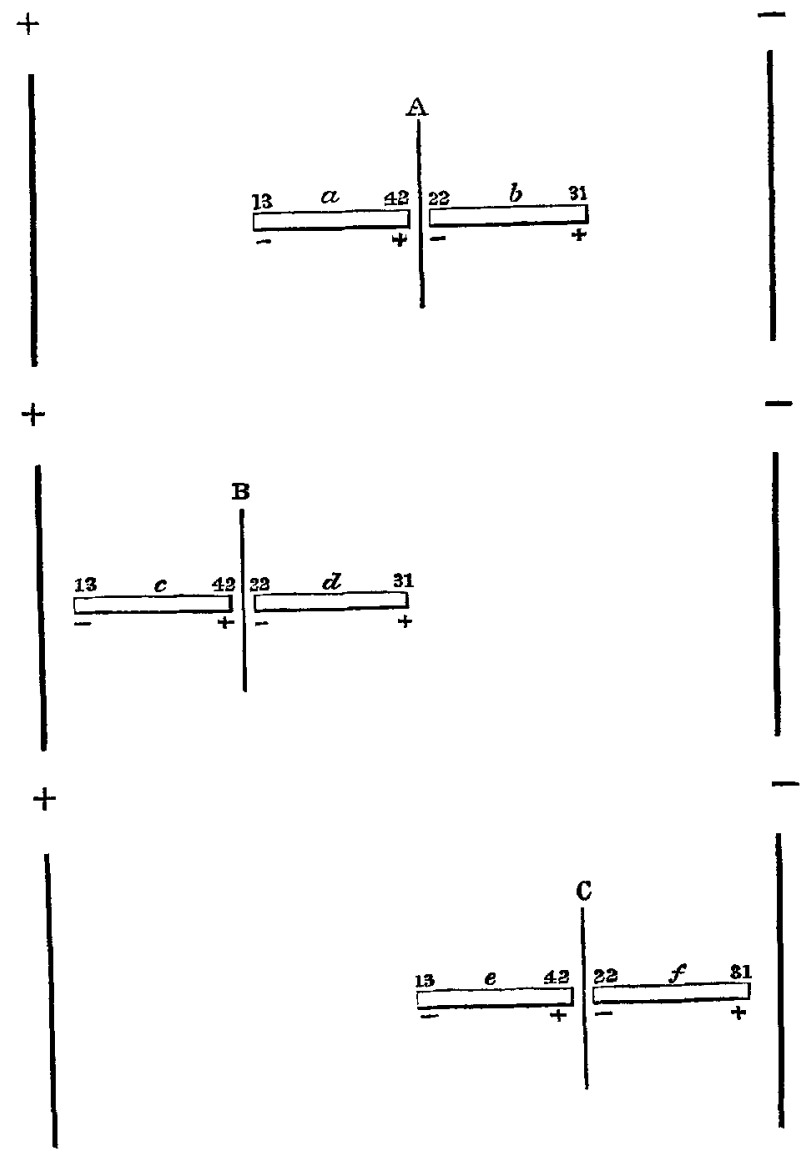


I might be allowed to point out that in a simple uniform field, such as would obtain were the ebonite plates absent, the magnitudes of like electrifications on analyzers in the positions indicated would have been absolutely identical. It will be observed, therefore, that the ebonite plates exerted an appreciable disturbing influence. It was of course to be foreseen that the direction of the lines of force would be changed, and the quantity of current flowing in the immediate neighbourhood of the analyzers would be diminished by the interposition of such a nonconducting plate; but the dissimilarity in the magnitudes of like electrifications, on the analyzers in the corresponding parts of the field relatively to the respective electrodes and sides of the plate, would appear to point to the existence of dissymmetry in those particular parts of the field, and, moreover, to show that this dissymmetry was produced by interference with the transmission of electricity by the electrolyte.

It was to be anticipated that, on reducing the amount of this interference, the dissymmetry would be reduced. Sach was found to be the case. On taking a smaller nonconducting plate the dissymmetry became less marked.

I would now point out, first, that the general effect of interposing a nonconducting plate as regards symmetry would appear to be similar to that produced by the spreading-out of the lines of force ; secondly, that the same extent of dissymmetry existed near the sides of the plate in very different parts of the field. This last fact shows that the phenomenon is independent of any direct action of the electrodes, attractive or otherwise.

In conclusion, I would remark that the dissymmetry pointed out in this paper is probably but a fresh example of the dissymmetry so common in ordinary electric discharges, and that the electrolytic field appears to possess advantages for the more complete examination of this very important subject. That the dissymmetry was real and primary, and not an apparent difference brought about by secondary action of the plates, would appear from the observations that the differences in the magnitudes of the like electrifications were found in corresponding parts of the field only when the lines of force were thrown out of their more direct course, and also from the observations that differences were found immediately the current passed into the electrolyte, when plates were used singly or together, when copper analyzers were employed and when the analyzers were of platinum (though in this case the electrifications only could be observed).

April 30, 1883. 